\title{
Productivity of different cropping systems as influenced by resource conservation techniques
}

\author{
Shaukat Ali*, A. M. Patel, Sangeeta Sharma ${ }^{1}$ and M. K. Man ${ }^{2}$ \\ Department of Agronomy, C.P. College of Agriculture, S. D. Agricultural University, SARDARKRUSHINAGAR \\ (GUJARAT) INDIA (Email : shaukatagro@gmail.com)
}

\begin{abstract}
The field experiment was conducted on loamy sand soils of Agronomy Instructional Farm, C. P. College of Agriculture, S. D. Agricultural University, Sardarkrushinagar (Gujarat) during the years 2012-13 and 2013-14 to study productivity of different cropping systems as influenced by resource conservation techniques. Cotton - summer pearlmillet cropping system was found significantly superior by recording higher pearlmillet equivalent yield and nutrients profitability, while, water productivity, water profitability and nutrients productivity were found higher under greengram + Kharif castor (relay) cropping system. Greengram - mustard - summer pearlmillet recorded the highest agro-energy. Residue incorporation secured top position by recording significantly the highest pearlmillet equivalent yield, water productivity, water profitability, nutrients productivity, nutrients profitability as well as agro-energy. The application of $75 \%$ RDN through inorganic fertilizer + $25 \%$ RDN through FYM recorded significantly the highest pearlmillet equivalent yield, water productivity, water profitability, nutrients productivity, nutrients profitability and agro-energy.
\end{abstract}

Key Words : Cropping system, Residue incorporation, FYM, Nutrients productivity, Nutrients profitability, Water productivity, Water profitability

View Point Article : Ali, Shaukat, Patel, A.M., Sharma, Sangeeta and Man, M.K. (2018). Productivity of different cropping systems as influenced by resource conservation techniques. Internat. J. agric. Sci., 14 (1) : 141-148, DOI:10.15740/HAS/IJAS/14.1/141-148.

Article History : Received : 17.05.2017; Revised : 22.11.2017; Accepted : 05.12.2017

\footnotetext{
* Author for correspondence (Present Address):

${ }^{1}$ Department of Clothing and Textile, Mahatma Jyoti Rao Phoole University, JAIPUR (RAJASTHAN) INDIA

${ }^{2}$ Department of Agronomy, School of Agriculture, Suresh Gyan Vihar University, JAIPUR (RAJASTHAN) INDIA
} 